\title{
Body mass index (BMI) and outcome of metastatic melanoma patients receiving targeted therapy and immunotherapy: a multicenter international retrospective study
}

\author{
Piotr Rutkowski (D , ${ }^{1}$ Alice Indini, ${ }^{2}$ Matilde De Luca, ${ }^{3}$ Barbara Merelli, ${ }^{2}$ \\ Anna Mariuk-Jarema, ${ }^{1}$ Pawel Teterycz, ${ }^{1}$ Pawel Rogala, ${ }^{1}$ Iwona Lugowska, ${ }^{1}$ \\ Bożena Cybulska-Stopa (10 , ${ }^{4}$ Alice Labianca, ${ }^{2}$ Lorenza Di Guardo, ${ }^{5}$ \\ Michele Del Vecchio, ${ }^{5}$ Jacopo Pigozzo, ${ }^{6}$ Giovanni Randon, ${ }^{5}$ Francesca Corti, ${ }^{5}$ \\ Carlo Alberto Tondini, ${ }^{2}$ Eliana Rulli, ${ }^{3}$ Mario Mandala (i) ${ }^{2,7}$
}

To cite: Rutkowski P, Indini A, De Luca M, et al. Body mass index (BMI) and outcome of metastatic melanoma patients receiving targeted therapy and immunotherapy: a multicenter international retrospective study. Journal for ImmunoTherapy of Cancer 2020;8:e001117. doi:10.1136/jitc-2020-001117

- Additional material is published online only. To view please visit the journal online (http://dx.doi.org/10.1136/jitc2020-001117).

ER and MM contributed equally.

ER and MM are joint senior authors.

Accepted 19 0ctober 2020

Check for updates

(c) Author(s) (or their employer(s)) 2020. Re-use permitted under CC BY-NC. No commercial re-use. See rights and permissions. Published by BMJ.

For numbered affiliations see end of article.

Correspondence to

Dr Mario Mandala;

mmandala@asst-pg23.it

\section{ABSTRACT}

Background Obesity is a risk factor for malignancy; however, its prognostic role in patients with metastatic melanoma is controversial. We aim to investigate the prognostic role of body mass index (BMI) in patients with metastatic melanoma receiving mitogen-activated pathway kinase inhibitors (MAPKi), immune checkpoint inhibitors (ICls) alone or their sequence.

Methods Data on patients with metastatic melanoma receiving $\geq 1$ line of systemic treatment were retrieved from prospectively collected databases. Progression-free survival (PFS) and overall survival (OS) were analyzed by means of multivariable stratified Cox regression models; disease control rate (DCR) was analyzed by multivariable stratified logistic regression models. Subgroup analyzes according to the type of treatments received, and in BRAF-mutated patients were pre-planned. All multivariable models included BMI, age, gender, American Joint Committee on Cancer stage, performance status, lactate dehydrogenase and treatment sequencing strategy as covariates.

Results Between November 2010 and November 2018, 688 patients from three Italian and two Polish centers were enrolled. 379 (57\%) patients had M1c/d disease, 273 $(41 \%)$ were female and the mean BMI was $27.1(\mathrm{SD}=4.9)$. Considering first-line treatment, 446 patients $(66.8 \%)$ received ICls and $222 \mathrm{MAPKi}$. No impact of BMI on OS was detected either considering the first line of ICls, or ICls sequencing ( $\mathrm{HR}=1.02,95 \% \mathrm{Cl}: 0.99$ to $1.05, \mathrm{p}=0.202$, and $\mathrm{HR}=1.02,95 \% \mathrm{Cl}: 0.99$ to $1.04, \mathrm{p}=0.237$, respectively). $\mathrm{A}$ late effect of $\mathrm{BMI}$ on $\mathrm{OS}$ was found in patients treated with MAPKi: for five units increment, a $51 \%$ of risk reduction at 18 months and a $76 \%$ of risk reduction at 30 months were observed. No significant effect of BMI on PFS and DCR was found in any of the subgroup analyzes.

Conclusion In patients with metastatic melanoma receiving ICls, there is no impact of $\mathrm{BMI}$ on DCR, PFS and $\mathrm{OS}$. The late prognostic effect of BMI in patients treated with MAPKi should be considered hypothesis generating and needs to be further investigated.

\section{INTRODUCTION}

Obesity is defined as a medical condition in which excess body fat accumulates to an extent that it may have a negative effect on global health. ${ }^{1}$ In the recent decades, the world has experienced a significant growth in obesity incidence, with an annual increase of more than $1 \%$ every year. ${ }^{2}$ According to the data from the National Health and Nutrition Examination Survey, it is estimated that more than $40 \%$ of adults are affected by obesity in the USA. ${ }^{23}$

Body mass index (BMI) is an easy measure to determine whether individuals are in healthy weight on the basis of their height (ie, weight $(\mathrm{kg})$ divided by the square of the person's height $\left.(\mathrm{m})\left(\mathrm{kg} / \mathrm{m}^{2}\right)\right)$. According to the WHO, individuals with a BMI $\geq 25 \mathrm{~kg} / \mathrm{m}^{2}$ are classified as being overweight; while, individuals with a BMI $>30 \mathrm{~kg} / \mathrm{m}^{2}$ are classified as obese. ${ }^{4}$ BMI strongly impacts on individuals' health, with an increase in mortality with growing degrees of overweight, as measured by BMI. ${ }^{4}$

There is a strong correlation between BMI and cancer's incidence and outcomes. ${ }^{45}$ Higher values of BMI correlate with an increased risk of developing various types of cancer, including breast, colorectal, uterine, as well as prostate cancers. ${ }^{45}$ Additionally, there are mixed findings regarding the impact of obesity on cancer patients' outcomes, with data demonstrating both a detrimental and protective effect, depending on the pathological context analyzed. ${ }^{67}$

Epidemiological studies in melanoma patients found that obesity is associated with 
Breslow thickness, suggesting a higher biological aggressiveness of melanoma in obese patients. ${ }^{8}$ Interestingly, data in mice models suggest that weight decrease can reverse the aggressiveness of melanoma. ${ }^{9}$ However, this evidence has not been demonstrated in humans. Recent accumulating evidence has suggested a positive correlation between increased BMI and efficacy of immune checkpoint inhibitors (ICIs), or mitogen-activated pathway kinase inhibitors (MAPKi) in metastatic melanoma. ${ }^{10-12}$ The pro-inflammatory status promoted by adipocytes might partly explain this so-called obesity paradox. ${ }^{11}$ However, the correlation between BMI and response to MAPKi and ICIs in metastatic melanoma is not fully understood, and the prognostic impact of BMI is still under investigation, ${ }^{10-16}$

Moreover, most studies evaluated a potential effect of BMI in patients treated either with MAPKi or ICIs. ${ }^{10-16}$ Indeed, due to the significant development in therapeutic strategies over the last years, treatment paradigm of metastatic melanoma now consists mostly of sequential treatment strategies. Real-world data reporting the effect of BMI and its variations (ie, BMI measured as a continuous rather than a fixed variable) over subsequent distinct lines of treatment are still lacking.

Here, we sought to investigate a possible association between BMI and outcome of metastatic melanoma patients receiving ICIs and MAPKi, by interrogating large international prospectively collected homogeneous databases.

\section{MATERIALS AND METHODS}

The study included all consecutive metastatic melanoma patients, diagnosed and treated with MAPKi and ICIs at Italian Melanoma Intergroup (IMI) and two Polish cancer centers from November 2010 to November 2018. Data were prospectively collected into specific databases, with information on demographics, previous medical history, diagnosis, surgical procedures, pathological features and follow-up.

Approval to conduct this study was obtained from IMI Institutional Review Board and local ethical committees.

\section{Statistical methods}

BMI at treatment initiation was calculated as weight $(\mathrm{kg})$ divided by the square of height $(\mathrm{m})$ and it was analyzed both as a continuous and categorical variable according to the standard WHO definitions: ${ }^{4}$ underweight (BMI $\left.<18.5 \mathrm{~kg} / \mathrm{m}^{2}\right)$, normal weight (18.5 to $\left.24.9 \mathrm{~kg} / \mathrm{m}^{2}\right)$, overweight (25 to $\left.29.9 \mathrm{~kg} / \mathrm{m}^{2}\right)$ and obese $\left(\geq 30 \mathrm{~kg} / \mathrm{m}^{2}\right)$. Patients without height or weight data available for BMI calculation were excluded from this analysis. The main objectives were to assess the association of BMI with progression-free survival (PFS) and overall survival (OS). An exploratory aim was to investigate the association of BMI with disease control rate (DCR, with stable disease (SD) $>6$ months). Disease evaluation during treatment followed the National Melanoma Treatment Program
(Polish centers) and the IMI (Italian centers) guidelines. Both guidelines recommend time schedules every 3 months until disease progression. PFS was defined as the time from the date of treatment start to the date of progression or death from any causes, whichever comes first. Patients who did not progress or die at the time of analysis were censored at their last disease assessment date. OS was defined as the time from treatment start to the date of death from disease (ie, melanoma specific survival). DCR was defined as the proportion of patients with a complete response (CR), a partial response (PR) or with a SD.

Patients with at least one evaluable endpoint were included in the present analysis. A possible different impact of BMI on prognosis according to treatment was evaluated by means of interaction test. A p value $<0.05$ was used to consider the interaction test as statistically significant, and therefore to analyze patients separately according to the treatment received. For PFS and DCR analysis, the treatment received were classified as MAPKi or ICIs. For the OS analysis, the treatment was categorized as MAPKi only, ICIs only, MAPKi followed by ICIs or ICIs followed by MAPKi. A further subgroup analysis was planned in patients with BRAFV600 mutation. Survival curves were estimated with the Kaplan-Meier method. PFS and OS were analyzed by means of Cox regression model and results were expressed as HR with their 95\% CI. The proportional hazard (PH) assumption was tested including in the model the interaction between time and covariate. In case of significant interaction ( $p$ value $<0.10$ ) hazards were considered non-proportional, therefore a time-varying coefficient was included in the final Cox model. DCR was analyzed by means of logistic regression models and expressed as OR with their 95\% CI. All multivariate models included as covariates age, gender, American Joint Committee on Cancer (AJCC) stage, performance status and lactate dehydrogenase $(\mathrm{LDH})$. In the subgroup of patients receiving ICIs, the type of ICI (anti-PD-1 or ipilimumab) and the $B R A F V 600$ status were also included as covariates, while in the subgroup of patients with BRAFV600 mutation, the type of therapy was also included as a covariate. All the fitted models were stratified according to the enrolling center. All analyzes were performed using the statistical analysis system (SAS), V.9.4. All statistical tests were two-sided and considered significant at $\mathrm{p}<0.05$ (unless otherwise specified).

\section{RESULTS}

Overall, 730 patients received a systemic therapy between November 2010 and November 2018. Sixty-two patients $(8.5 \%)$ were excluded from the analyzes: 50 due to missing data on BMI, 11 received neither ICIs nor MAPKi (BRAF and/or MEK inhibitors), and data on main endpoints were missing for one patient. Among 668 patients, 129 were enrolled in Bergamo, 115 in Milan, 77 in Padua, 162 in Krakow and 185 in Warsaw. Overall, 659 (98.7\%), 653 $(97.8 \%)$ and $642(96.1 \%)$ were included in OS, PFS and DCR analysis, respectively. 
Table 1 Demographic and clinical characteristics of the enrolled population by first-line treatment

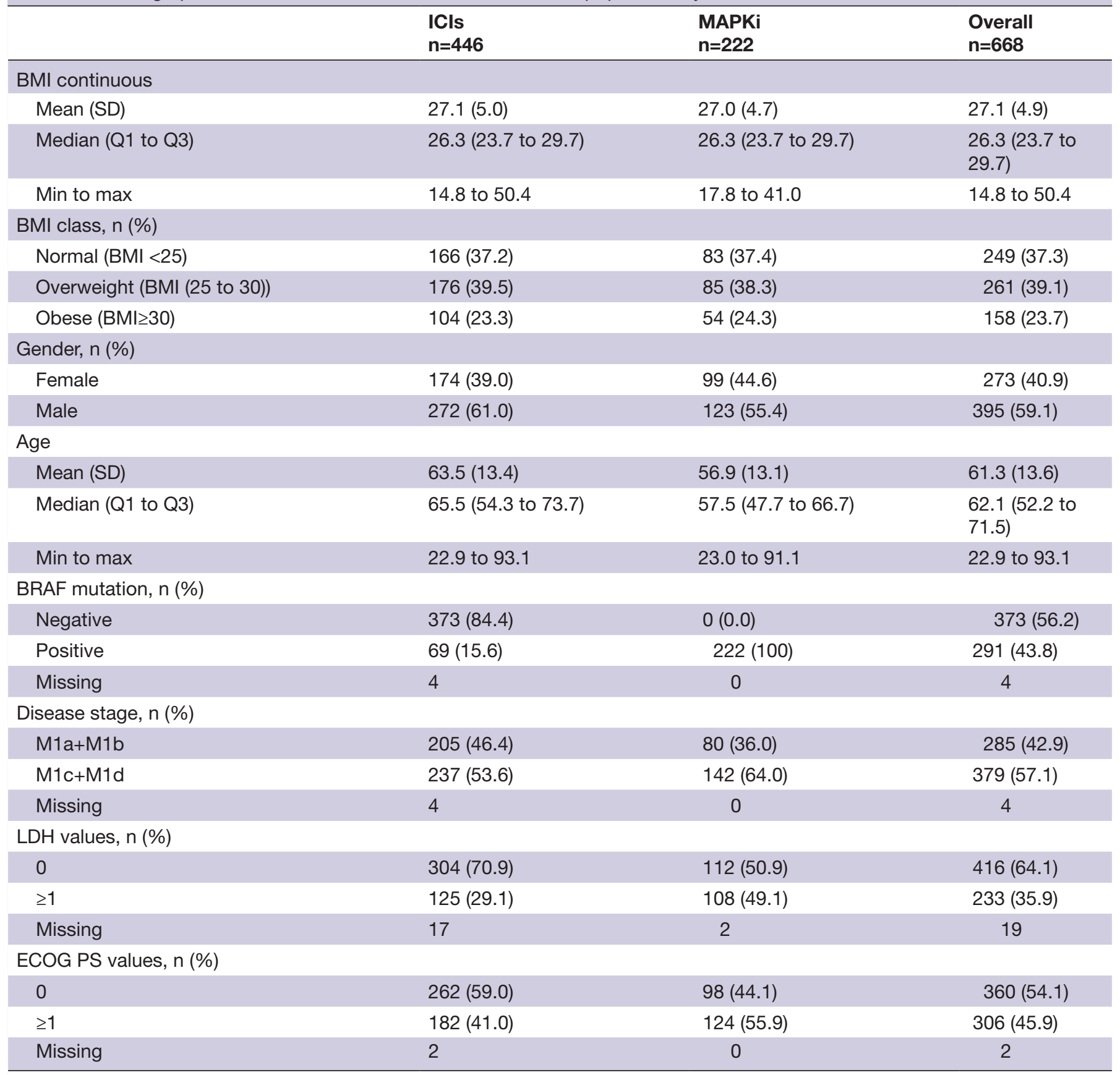

BMI, body mass index; ECOG, Eastern Cooperative Oncology Group Performance Status; ICls, immune checkpoint inhibitors; LDH, lactate dehydrogenase; MAPKi, mitogen activated pathway kinase inhibitors; Max, maximum; Min, minimum; n, number of subjects; $Q$, quartile.

Table 1 summarizes patients' characteristics according to the first line of treatment. The mean BMI was 27.1 ( $\mathrm{q} 1$ to q3: 23.7 to 29.7$)$. In total, $9(1.3 \%), 240(35.9 \%), 261$ $(39.1 \%)$ and $158(23.7 \%)$ patients were categorized as underweight, normal weight, overweight and obese, respectively. The mean age was 61.3 years ( $\mathrm{q} 1$ to $\mathrm{q} 3: 52.2$ to 71.5 ); $273(40.9 \%)$ were female and $373(56.2 \%)$ were BRAFV600 wild type; BRAFV600 status was unknown for four patients.

All the enrolled patients had metastatic (stage IV) melanoma: 110 (16.6\%) M1a, 175 (26.4\%) M1b, 267 (40.2) M1c, and $112(16.9 \%)$ M1d according to the AJCC VIII classification; four patients had undetermined disease stage. Considering the first line treatment, 446 patients $(66.8 \%)$ received ICIs and 222 MAPKi. Among 286 patients harboring BRAFV600 mutation, 67 (23\%) received ICIs and $219(77 \%)$ received MAPKi as first-line treatment.

\section{Impact of BMI on overall survival}

With a median follow-up of 42.5 months, $438(66.5 \%)$ out of 659 patients died. Overall, $417(63.3 \%)$ consecutive patients received ICIs alone, $106(16.1 \%)$ MAPKi alone, $113(17.1 \%)$ MAPKi followed by ICIs and 23 (3.5\%) ICIs 
Table 2 Multivariable Cox model for overall survival immunotherapy alone subset

\begin{tabular}{|c|c|c|c|}
\hline$n=393$ & $\begin{array}{l}P \text { value of } \\
\text { variable }\end{array}$ & HR $(95 \%$ Cl) & $\begin{array}{l}P \text { value of } \\
\text { contrasts }\end{array}$ \\
\hline BMI & 0.202 & 1.02 (0.99 to 1.05$)$ & - \\
\hline Gender & 0.922 & & \\
\hline Female & & Reference & \\
\hline Male & & 1.01 (0.77 to 1.33$)$ & 0.922 \\
\hline BRAF mutation & $0.034^{*}$ & & \\
\hline Negative & & Reference & \\
\hline Positive & & 0.56 (0.32 to 0.96$)$ & $0.034^{*}$ \\
\hline Disease stage & 0.114 & & \\
\hline $\mathrm{M} 1 \mathrm{a}+\mathrm{M} 1 \mathrm{~b}$ & & Reference & \\
\hline $\mathrm{M} 1 \mathrm{c}+\mathrm{M} 1 \mathrm{~d}$ & & 1.25 (0.95 to 1.66$)$ & 0.114 \\
\hline LDH value & $<0.001^{*}$ & & \\
\hline 0 & & Reference & \\
\hline$\geq 1$ & & 1.71 (1.28 to 2.28$)$ & $<0.001^{*}$ \\
\hline ECOG PS value & $<0.001^{*}$ & & \\
\hline 0 & & Reference & \\
\hline$\geq 1$ & & 2.11 (1.47 to 3.03 ) & $<0.001^{*}$ \\
\hline Age & 0.700 & 1.00 (0.99 to 1.01$)$ & - \\
\hline $\begin{array}{l}\text { Type of first-line } \\
\text { ICls }\end{array}$ & 0.514 & & \\
\hline Anti-PD1 & & Reference & \\
\hline Ipilimumab & & 1.14 (0.77 to 1.69$)$ & 0.514 \\
\hline
\end{tabular}

${ }^{*}$ For $\mathrm{LDH}$, the $\mathrm{PH}$ assumption is not satisfied.

BMI, body mass index; ECOG PS, Eastern Cooperative Oncology Group performance status; ICI, immune checkpoint inhibitor; $\mathrm{LDH}$, lactate dehydrogenase; $\mathrm{PH}$, proportional hazard.

followed by MAPKi. The test of interaction between BMI and the type of therapy was statistically significant $(\mathrm{p}=0.009)$, suggesting that the impact of BMI on survival was different in patients receiving ICIs versus MAPKi, or a combination of both therapies.

At a median follow-up of 38.8 months, 246 out of 417 patients $(59 \%)$ who received ICIs alone died, while the remaining $171(41 \%)$ were alive. The median OS was 24.6 months (q1 to q3: 8.4 to not reached). Three hundred and thirty-one patients (79.4\%) and $86(20.6 \%)$ patients received an anti-PD1 and ipilimumab as first-line treatment, respectively. Considering all lines of immunotherapy, 23 patients $(5.5 \%)$ received ipilimumab alone, 281 (67.4\%) anti-PD1 alone and 113 (27.1\%) ICIs sequence. Considering the ICIs sequence, 63 out of $113(55.8 \%)$ patients received ipilimumab before, while $50(44.2 \%)$ received anti-PD1 as first-line therapy.

Table 2 summarizes results of the multivariable Cox $\mathrm{PH}$ model in patients receiving ICIs alone. No impact of BMI on OS was detected either considering the first line of ICIs, or the ICIs' sequence (HR=1.02, 95\% CI: 0.99 to $1.05, \mathrm{p}=0.202$, and $\mathrm{HR}=1.02,95 \%$ CI: 0.99 to $1.04, \mathrm{p}=0.237$,
Table 3 Multivariable Cox model for overall survival in targeted therapy alone subset

\begin{tabular}{|c|c|c|c|}
\hline$n=104$ & $\begin{array}{l}P \text { value of } \\
\text { variable }\end{array}$ & HR $(95 \% \mathrm{Cl})$ & $\begin{array}{l}P \text { value of } \\
\text { contrasts }\end{array}$ \\
\hline BMI & 0.310 & 0.97 (0.91 to 1.03 ) & - \\
\hline Gender & 0.980 & & \\
\hline Female & & Reference & \\
\hline Male & & 1.01 (0.63 to 1.61$)$ & 0.980 \\
\hline Disease stage & 0.163 & & \\
\hline$M 1 a+M 1 b$ & & Reference & \\
\hline $\mathrm{M} 1 \mathrm{c}+\mathrm{M} 1 \mathrm{~d}$ & & 1.53 (0.84 to 2.80$)$ & 0.163 \\
\hline LDH value & 0.100 & & \\
\hline 0 & & Reference & \\
\hline$\geq 1$ & & 1.51 (0.92 to 2.47$)$ & 0.100 \\
\hline ECOG PS value & $0.008^{*}$ & & \\
\hline 0 & & Reference & \\
\hline$\geq 1$ & & $2.42(1.26$ to 4.65$)$ & $0.008^{*}$ \\
\hline Age & 0.927 & 1.00 (0.98 to 1.02$)$ & - \\
\hline
\end{tabular}

For BMI, the $\mathrm{PH}$ assumption is not satisfied.

BMI, body mass index; ECOG PS, Eastern Cooperative Oncology Group performance status; LDH, lactate dehydrogenase; $\mathrm{PH}$, proportional hazard.

respectively). Similar results were obtained considering the BMI as categorical parameter (online supplemental table 1). The statistical significance of the interaction between BMI and gender was evaluated to assess a different effect of BMI on OS according to gender. In the cohort of ICIs patients, the $\mathrm{p}$ value of interaction between gender and BMI was not statistically significant $(\mathrm{p}=0.160)$.

At a median follow-up of 47.2 months, among the 106 patients treated with MAPKi alone, 82 died $(77.4 \%)$. The median OS was 9.2 months (q1 to q3: 5.7 to 32.2). Table 3 summarizes the results of the multivariable Cox PH model in the cohort of patients treated with MAPKi alone. No impact of BMI on OS was detected. However, non-PH was found ( $p$ value interaction $=0.031$ ), accordingly an adjusted time-varying HR was estimated for BMI (online supplemental table 2) for results of the corresponding Cox model. As illustrated in figure 1 , the risk of death related to BMI increase lowered with time. Since the observed trend became significant after 13 months, a 'late' effect of BMI on OS was found. Specifically, for five units increment in BMI, a $51 \%$ risk reduction of death was observed at 18 months (ie, when $31(29.2 \%)$ patients were still at risk of dying) and a $76 \%$ risk reduction was reached at 30 months (ie, with $24(22.6 \%)$ patients at risk). Superimposable results were obtained considering the BMI as a categorical parameter, in particular for obese versus normal weight subjects (online supplemental table 3). Moreover, also in the cohort of patients treated with MAPKi, no statistically significant interaction between gender and BMI was found ( $p$ value interaction=0.472). 


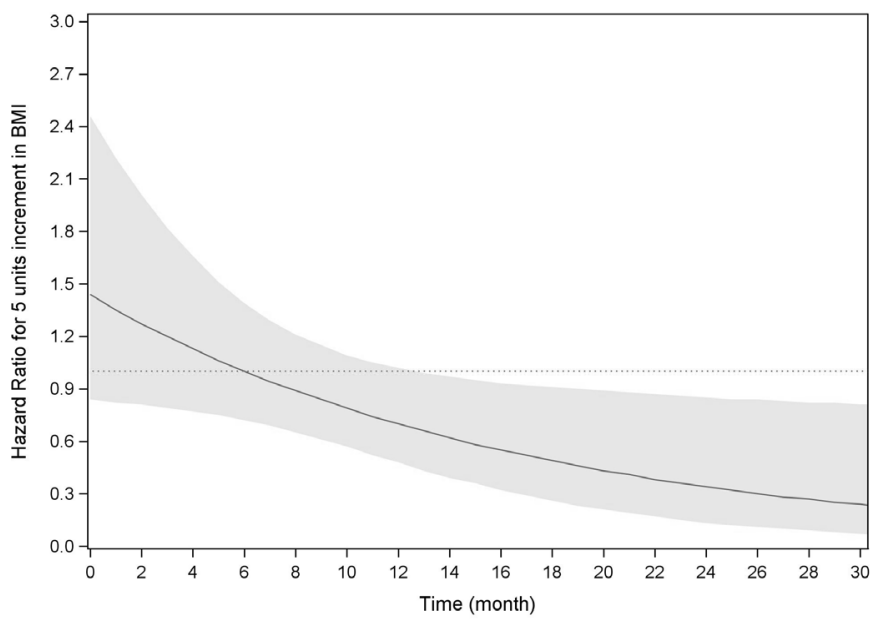

\begin{tabular}{ccccc}
\hline time & p-value & HR (95\% CI) & $\begin{array}{c}5 \text { units increment on BMI } \\
\text { HR (95\% CI) }\end{array}$ & Patients at risk \\
\hline 0 & 0.188 & $1.08(0.97-1.20)$ & $1.44(0.84-2.46)$ & $106(100 \%)$ \\
6 & 0.997 & $1.00(0.94-1.07)$ & $1.00(0.73-1.39)$ & $76(71.7 \%)$ \\
12 & 0.062 & $0.93(0.86-1.00)$ & $0.70(0.48-1.02)$ & $46(43.4 \%)$ \\
13 & 0.045 & $0.92(0.85-1.00)$ & $0.66(0.43-0.99)$ & $44(41.5 \%)$ \\
18 & 0.024 & $0.87(0.76-0.98)$ & $0.49(0.26-0.91)$ & $31(29.2 \%)$ \\
24 & 0.021 & $0.81(0.67-0.97)$ & $0.34(0.13-0.85)$ & $25(23.6 \%)$ \\
30 & 0.022 & $0.75(0.58-0.96)$ & $0.24(0.07-0.81)$ & $24(22.6 \%)$ \\
\hline
\end{tabular}

Figure 1 Time varying HR for five units increment of body mass index over time. Overall survival in target therapy alone subset.

At a median follow-up of 67 months, 98 out of 113 patients $(86.7 \%)$ treated with MAPKi followed by ICIs died, while $15(13.3 \%)$ were alive. The median OS was 16.7 months (q1-q3: 11.4-39.1). Seventy-two (63.7\%) patients received anti-PD1, while 41 (36.3\%) received ipilimumab as first-line ICI. Among 15 patients $(13.3 \%)$ who received more than one line of ICIs, two patients received anti-PD1 followed by ipilimumab, while the others received ipilimumab followed by anti-PD1. The effect of BMI on OS was not statistically significant ( $\mathrm{HR}=1.00,95 \%$ CI: 0.96 to 1.05 , $\mathrm{p}=0.896$ ) (online supplemental table 4), similar results were obtained considering BMI as a categorical variable (online supplemental table 5). No statistically significant interaction between BMI and gender was found ( $p$ value of interaction $=0.832$ ).

At a median follow-up of 47.2 months, 207/286 (72.4\%) patients with BRAFV600 mutation died. The median OS was 16.6 months ( $q 1$ to q3: 8.6 to 53.5). The majority of these patients received a MAPKi: $106(37.1 \%)$ MAPKi alone, $113(39.5 \%)$ MAPKi followed by ICIs and $23(8 \%)$ ICIs followed by MAPKi; 44 subjects $(15.4 \%)$ were treated with ICIs only. The adjusted effect of BMI on OS was not statistically significant (HR=0.97, 95\% CI: 0.94 to 1.01 , $\mathrm{p}=0.108$ ) (online supplemental tables 6 and 7) for continuous and categorical BMI. The interaction between BMI and gender was not statistically significant $(\mathrm{p}=0.937)$.

Figure 2 shows the Kaplan-Meier curves for OS in the whole population, according to BMI categories.

\section{Progression-free survival}

Considering the whole population, at a median PFS of 8.3 months (q1: 3.7 to q3: 26.6), $516(79.1 \%)$ patients died or experienced disease progression (83 patients had disease progression, 52 died without disease progression and 381 progressed and then died); $435(66.6 \%)$ and 218 (33.4\%) patients received ICIs and MAPKi as first-line treatment, respectively.

Among 435 patients treated with ICIs, 341 (78.4\%) received an anti-PD1 and the remaining 94 (21.6\%)

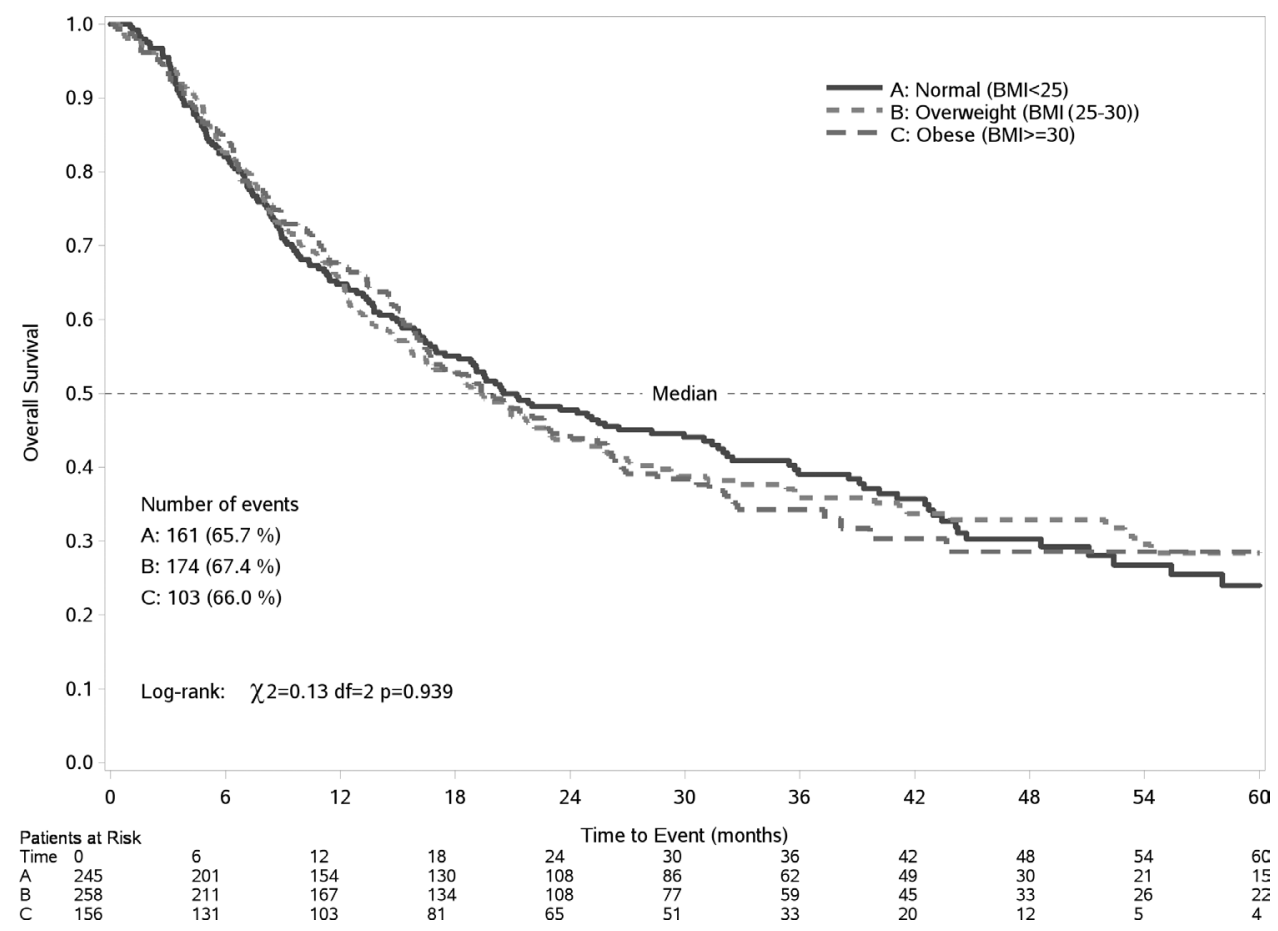

Figure 2 Kaplan-Meier estimate of overall survival in the whole population by BMI categories. BMI, body mass index. 
ipilimumab. No statistically significant interaction was found for BMI and type of immunotherapy $(\mathrm{p}=0.132)$. Median PFS was 8.6 months (q1 to q3: 3.0 to 40.1), 322 (74.2\%) subjects experienced a PFS event (progression or death). No significant effect of BMI on PFS was shown (adjusted HR=1.00, 95\% CI: 0.98 to 1.03, $\mathrm{p}=0.732$ ) (online supplemental tables 8 and 9) for continuous and categorical BMI. The interaction between BMI and gender was not statistically significant $(\mathrm{p}=0.432)$. A sensitivity analysis, which included only patients treated with anti-PD1 showed similar results $(\mathrm{n}=341$, adjusted $\mathrm{HR}=1.01,95 \%$ CI: 0.98 to $1.04, \mathrm{p}=0.462$ ) (online supplemental table 10).

Considering the 218 subjects who received MAPKi, 194 patients $(89.0 \%)$ died or experienced disease progression, with a median PFS of 7.6 months (q1 to q3: 4.5 to 14.2). No effect of BMI was detected, with an adjusted HR of 0.98 (95\% CI: 0.94 to $1.01, \mathrm{p}=0.194$ ) (online supplemental tables 11 and 12) for continuous and categorical BMI. The interaction between BMI and gender was not statistically significant $(\mathrm{p}=0.317)$.

Among the 285 patients with BRAFV600 mutation, 241 (84.6\%) patients progressed or died. The median PFS was 8.2 months (q1 to q3: 4.3 to 19.5). No effect of BMI was detected (adjusted HR=0.97, 95\% CI: 0.94 to 1.00 , $\mathrm{p}=0.067$ ) (online supplemental tables 13 and 14) for continuous and categorical BMI. The interaction between BMI and gender was not statistically significant $(\mathrm{p}=0.526)$.

The Kaplan-Meier curves for PFS in the whole study population, according to BMI categories, are reported in online supplemental figure 1 .

\section{Disease control rate}

Among 642 patients evaluable for DCR, 138 (21.5\%) experienced progressive disease (PD) as best response, 75 (11.7\%) had CR, 208 (32.4\%) PR and 221 (34.4\%) SD (for at least 6 months). Considering the first-line therapy, $426(66.4 \%)$ patients received ICIs and $216(33.6 \%)$ MAPKi.

Among the 426 patients who received ICIs, 118 (27.7\%) had PD as best response, 51 (12.0\%) CR, 98 (23.0\%) PR and $159(37.3 \%)$ SD The majority of patients received anti-PD1 $(77.7 \%)$ and the remaining ipilimumab. No interaction between BMI and type of immunotherapy was detected ( $p=0.960)$. No effect of BMI on DCR was shown (adjusted $\mathrm{OR}=0.98,95 \%$ CI 0.93 to $1.03, \mathrm{p}=0.432$ ) (online supplemental tables 15 and 16) for continuous and categorical BMI. The interaction between BMI and gender was not statistically significant ( $\mathrm{p}$ value of interaction $=0.841$ ).

Considering the 216 subjects treated with MAPKi, 20 (9.3\%) had PD, 24 (11.1\%) CR, $110(50.9 \%)$ PR and $62(28.7 \%)$ SD Even in this subgroup, no effect of BMI on DCR was shown (adjusted OR=1.04, 95\% CI 0.93 to 1.17, $\mathrm{p}=0.499$ ) (online supplemental tables 17 and 18) for continuous and categorical BMI. The interaction between BMI and gender was not statistically significant ( $p$ value for interaction $=0.586$ ) .

Among the 642 patients evaluable for response to firstline treatment, $356(55.8 \%)$ were BRAFV600 wild-type,
$282(44.2 \%)$ were BRAFV600 mutated and four had unknown BRAF mutational status. In the BRAFV600mutant subgroup, $38(13.5 \%)$ had PD as best response, $31(11.0 \%)$ CR, 125 (44.3\%) PR and 88 (31.2\%) SD. No effect of BMI on DCR was shown (adjusted $\mathrm{OR}=1.03$, $95 \%$ CI 0.94 to $1.12, \mathrm{p}=0.578$ ) (online supplemental tables 19 and 20) for continuous and categorical BMI. The interaction between BMI and gender was not statistically significant ( $\mathrm{p}$ value for interaction $=0.920$ ).

\section{DISCUSSION}

There is a complex relationship between obesity and melanoma. Preclinical and clinical data showed a correlation between obesity and Breslow thickness, suggesting a higher biologic aggressiveness of melanoma in obese patients. ${ }^{8}$ Paradoxically, some recent studies have reported a direct correlation between BMI and response to ICIs and MAPKi in metastatic melanoma, highlighting that the increase in BMI may improve the activity and the efficacy of ICIs and MAPKi. ${ }^{10-12}$ The pro-inflammatory status promoted by adipocytes might partly explain this so-called obesity paradox. ${ }^{11}$

In this context, our study is timely and potentially relevant to better understand the impact of obesity and its relationship with the activity and the efficacy of new systemic strategies in advanced melanoma. Prevalence of obesity was quite high in our study population (median BMI=27.1), which is somehow similar to the values reported in US population studies, supporting the finding that cachexia is infrequent in this subset of patients. ${ }^{10-12}$ Our study is based on data derived from routine activity and, accordingly, a relevant proportion of patients received sequential treatments, mirroring the modern approach in metastatic melanoma patients. As so, the subgroup of $B R A F$ positive patients receiving first line targeted therapy may have had a more extensive disease, a worse performance status, higher LDH levels and younger age, compared with BRAF-positive patients receiving immunotherapy as first-line treatment.

By interrogating prospectively collected database and including patients treated in the context of routine activity, we were not able to find any correlation between BMI and response rate or PFS in melanoma patients receiving ICIs and/or MAPKi. Importantly, in our study, BMI was evaluated as a continuous variable as well as a categorical parameter in multivariate analysis, after adjusting for all the established prognostic factors.

In both the ICI and the MAPKi cohorts, the estimated effects of BMI on survival were not statistically significant at early times. At a median follow-up of 42.5 months, considering patients receiving ICIs, we did not find BMI to have a detrimental late effect on survival. In patients treated with ICIs, the PH assumption for BMI was satisfied, implying the absence of a late effect of BMI on survival. Our data support this result both by analyzing ICI as monotherapy and sequential strategy. Conversely, for the MAPKi cohort, only 44 out of 106 patients (41.5\%) 
had a survival time $\geq 13$ months, which is the time point from which the impact of BMI was statistically significant. Considering this, we cannot exclude that such result can partly be explained by an early selection of patients with poor prognostic characteristics (eg, presence of brain metastases).

Overall, our data suggest that further research is needed to elucidate the relationship between obesity and melanoma, and we think that the scientific community should consider several important issues and weaknesses of the data so far reported prior to drawing conclusions that could potentially influence patient care.

There are four important issues to consider while examining results available so far on the correlation between obesity and new systemic therapies in melanoma. First, clinical results have not shown a unique correlation between BMI and immunotherapy, with inconsistent results through populations receiving either anti-CTLA4 or anti-PD1. Wang et al, ${ }^{17}$ Naik et $a l^{11}$ and Cortellini et $a l^{18}$ showed a significantly positive association between BMI and survival in melanoma patients treated with anti-PD1 antibody; Richtig et $a l^{12}$ showed a positive association in patients treated with anti-CTLA4; McQuade $e t a l^{10}$ showed a positive association for both patients receiving anti-PD1 and anti-CTLA4 antibodies; Donnelly et $a l^{19}$ showed a positive association in patients treated with combination of anti-CTLA-4 and anti-PD1, but not with anti-CTLA-4 or anti-PD1 alone. The reasons for such inconsistent results partly lie in the heterogeneity of study populations, dosing of ICIs as weight-based versus fixed, and the absence of pharmacokinetic control in analyzing the correlation between BMI and systemic treatments. ${ }^{19}$

Second, the studies adjusted their analyzes using different covariates and used distinct statistical models to assess the associations.

Third, all the studies included different patients' cohort, and different time schedule evaluation during systemic treatments.

Fourth, BMI as described by the WHO classification does not recapitulate the right body composition. In view of this limitation, some authorities advocate a definition of obesity based on percentage of body fat. For men, a percentage of body fat greater than $25 \%$ defines obesity, with $21 \%$ to $25 \%$ being borderline. For women, over $33 \%$ defines obesity, with $31 \%$ to $33 \%$ being borderline. Other indices include the four standard skin thicknesses (ie, subscapular, triceps, biceps and suprailiac) and various anthropometric measures, of which waist and hip circumferences are the most important. The current standard techniques for measuring visceral fat volume are abdominal CT and MRI techniques, and, more recently, bioelectrical impedance. Translational studies are needed with longitudinal biopsies, in order to better understand the biological correlates linked to obesity.

The strengths of our study include (i) the large cohort of patients included; (ii) data prospectively collected into specific databases with information regarding demographics, patient clinical history, diagnosis, systemic therapy, objective response, PFS and OS; (iii) the longest follow-up to date in this subset of patients, thus allowing to investigate specific survival endpoints.

We are aware of some limitations, including the retrospective nature of our analysis which cannot exclude patient enrollment bias. Furthermore, we included only BMI value at baseline and no longitudinal assessment has been performed, although cachexia in melanoma is rare at disease progression. A potential danger in our analysis is that analyzing data at late time points could be less reliable, due to the lower number of patients surviving at late time points.

In conclusion, our study suggests that it is important to better and further investigate BMI as a potential biomarker for a positive clinical outcome of patients treated with ICIs or MAPKi. It is imperative to not overemphasize associations found in some cohort studies considering the inconsistency of the trials so far reported and more translational longitudinal studies are needed in order to clarify the so-called obesity paradox in the context of new systemic strategies in metastatic melanoma.

\section{Author affiliations}

${ }^{1}$ Department of Soft Tissue/Bone Sarcoma and Melanoma, Maria Sklodowska-Curie National Research Institute of Oncology in Warsaw, Warszawa, Poland ${ }^{2}$ Oncology and Hematology, ASST Papa Giovanni XXIII, Bergamo, Italy ${ }^{3}$ Istituto di Ricerche Farmacologiche Mario Negri Sede di Milano, Milano, Lombardia, Italy

${ }^{4}$ Maria Sklodowska-Curie Institute of Oncology Krakow, Krakow, Poland

${ }^{5}$ Fondazione IRCCS Istituto Nazionale dei Tumori, Milano, Lombardia, Italy ${ }^{6}$ Istituto Oncologico Veneto Istituto di Ricovero e Cura a Carattere Scientifico, Padova, Veneto, Italy

${ }^{7}$ University of Perugia, Perugia, Italy

Contributors All authors provided a significant contribution to the present manuscript.

Funding The authors have not declared a specific grant for this research from any funding agency in the public, commercial or not-for-profit sectors.

Competing interests No, there are no competing interests.

Patient consent for publication Not required.

Ethics approval Approval to conduct this study was obtained from the Italian Melanoma Intergroup (IMI) Institutional Review Board and local ethical committees. All patients involved in this study gave their written consent for participation and use of information for medical and scientific purposes.

Provenance and peer review Not commissioned; externally peer-reviewed.

Data availability statement All data relevant to the study are included in the article or uploaded as supplementary information. Additional data are available upon reasonable request.

Supplemental material This content has been supplied by the author(s). It has not been vetted by BMJ Publishing Group Limited (BMJ) and may not have been peer-reviewed. Any opinions or recommendations discussed are solely those of the author(s) and are not endorsed by BMJ. BMJ disclaims all liability and responsibility arising from any reliance placed on the content. Where the content includes any translated material, BMJ does not warrant the accuracy and reliability of the translations (including but not limited to local regulations, clinical guidelines, terminology, drug names and drug dosages), and is not responsible for any error and/or omissions arising from translation and adaptation or otherwise.

Open access This is an open access article distributed in accordance with the Creative Commons Attribution Non Commercial (CC BY-NC 4.0) license, which permits others to distribute, remix, adapt, build upon this work non-commercially, and license their derivative works on different terms, provided the original work is 
properly cited, appropriate credit is given, any changes made indicated, and the use is non-commercial. See http://creativecommons.org/licenses/by-nc/4.0/.

\section{ORCID iDs}

Piotr Rutkowski http://orcid.org/0000-0002-8920-5429

Bożena Cybulska-Stopa http://orcid.org/0000-0002-0975-850X

Mario Mandala http://orcid.org/0000-0001-8846-8959

\section{REFERENCES}

1 Engin $A$. The definition and prevalence of obesity and metabolic syndrome. Adv Exp Med Biol 2017;960:1-17.

2 National Health and Nutrition Examination Survey. National Obesity Rates \& Trends. Available: https://www.stateofobesity.org/obesityrates-trends-overview/ [Accessed 8 Dec 2019].

3 Harvard. School of public health. The nutrition source. an epidemic of obesity: U.S. obesity trends. Available: https://www.hsph.harvard edu/nutritionsource/an-epidemic-of-obesity/ [Accessed 8 Dec 2019].

4 World Health organization (who), global health Observatory (GHO) data. Available: https://www.who.int/gho/ncd/risk_factors/bmi_text/ en/ [Accessed 8 Dec 2019].

5 Vernaci G, Dieci MV, Manfrin S, et al. Bmi is an independent prognostic factor for late outcome in patients diagnosed with early breast cancer: a landmark survival analysis. Breast 2019;47:77-84.

6 Schlick K, Magnes T, Ratzinger L, et al. Novel models for prediction of benefit and toxicity with Folfirinox treatment of pancreatic cancer using clinically available parameters. PLoS One 2018;13:e0206688.

7 Lennon H, Sperrin M, Badrick E, et al. The obesity paradox in cancer: a review. Curr Oncol Rep 2016;18:56.

8 Skowron F, Bérard F, Balme B, et al. Role of obesity on the thickness of primary cutaneous melanoma. J Eur Acad Dermatol Venereol 2015;29:262-9.
9 Brandon EL, Gu J-W, Cantwell L, et al. Obesity promotes melanoma tumor growth: role of leptin. Cancer Biol Ther 2009;8:1871-9.

10 McQuade JL, Daniel CR, Hess KR, et al. Association of bodymass index and outcomes in patients with metastatic melanoma treated with targeted therapy, immunotherapy, or chemotherapy: a retrospective, multicohort analysis. Lancet Oncol 2018;19:310-22.

11 Naik GS, Waikar SS, Johnson AEW, et al. Complex inter-relationship of body mass index, gender and serum creatinine on survival: exploring the obesity paradox in melanoma patients treated with checkpoint inhibition. J Immunother Cancer 2019;7:89.

12 Richtig G, Hoeller C, Wolf M, et al. Body mass index may predict the response to ipilimumab in metastatic melanoma: an observational multi-centre study. PLoS One 2018;13:e0204729.

13 Young A, Quach HT, Davis EJ, et al. Impact of body composition on outcomes from anti-programmed death-1 (PD-1) treatment. J Clin Oncol 2019;37:9516.

14 Hemadri A, Lin H, Lin Y, et al. Association of baseline body mass index (BMI) with response and survival in patients (PTS) with advanced melanoma (MEL) receiving PD-1 inhibitors. J Clin Oncol 2019;37:9579.

15 Donnelly DM, Bajaj S, Zhong J, et al. The relationship between obesity and immunotherapy: It's complicated. JCO 2019;37:9562.

16 Desai A, Parmar HV, Hegde UP. obesity and metastatic melanoma: A national perspective from the pre-immune checkpoint inhibitor era. JCO 2019;37:e21032-e.

17 Wang Z, Aguilar EG, Luna Jl, et al. Paradoxical effects of obesity on $T$ cell function during tumor progression and PD-1 checkpoint blockade. Nat Med 2019;25:141-51.

18 Cortellini A, Bersanelli M, Buti S, et al. A multicenter study of body mass index in cancer patients treated with anti-PD-1/PD-L1 immune checkpoint inhibitors: when overweight becomes favorable. $J$ Immunother Cancer 2019;7:57.

19 Donnelly D, Bajaj S, Yu J, et al. The complex relationship between body mass index and response to immune checkpoint inhibition in metastatic melanoma patients. J Immunother Cancer 2019;7:222. 